\title{
Clinical and imaging features of myeloid sarcoma: a German multicenter study
}

\author{
Hans-Jonas Meyer ${ }^{1 *}$ (D), Wolfram Pönisch ${ }^{2}$, Stefan Andreas Schmidt ${ }^{3}$, Susanne Wienbeck ${ }^{4}$, Friederike Braulke ${ }^{5}$, \\ Dominik Schramm ${ }^{6}$ and Alexey Surov ${ }^{1}$
}

\begin{abstract}
Background: Myeloid sarcoma (MS), also known as chloroma, is an extramedullary manifestation of malignant primitive myeloid cells. Previously, only small studies investigated clinical and imaging features of MS. The purpose of this study was to elucidate clinical and imaging features of MS based upon a multicenter patient sample.

Methods: Patient records of radiological databases of 4 German university hospitals were retrospectively screened for MS in the time period 01/2001 and 06/2019. Overall, 151 cases/76 females (50.3\%) with a mean age of $55.5 \pm$ 15.1 years and 183 histopathological confirmation or clinically suspicious lesions of MS were included into this study. The underlying hematological disease, localizations, and clinical symptoms as well as imaging features on CT and MRI were investigated.
\end{abstract}

Results: In 15 patients (9.9\% of all 151 cases) the manifestation of MS preceded the systemic hematological disease. In 43 cases (28.4\%), first presentation of MS occurred simultaneously with the initial diagnosis of leukemia, and 92 (60.9\%) patients presented MS after the initial diagnosis. In 37 patients (24.5\%), the diagnosis was made incidentally by imaging. Clinically, cutaneous lesions were detected in 35 of 151 cases (23.2\%). Other leading symptoms were pain $(n=28 / 151,18.5 \%)$, neurological deficit $(n=27 / 151,17.9 \%)$, swelling $(n=14 / 151,9.3 \%)$ and dysfunction of the affected organ ( $n=10 / 151,6.0 \%)$. Most commonly, skin was affected $(n=30 / 151,16.6 \%)$, followed by bone $(n=29 /$ $151,16.0 \%)$ and lymphatic tissue $(n=21 / 151,11.4 \%)$. Other localizations were rare. On $C T$, most lesions were homogenous. On T2-weighted imaging, most of the lesions were hyperintense. On T1-weighted images, MS was hypointense in $n=22 / 54$ (40.7\%) and isointense in $n=30 / 54$ (55.6\%). A diffusion restriction was identified in most cases with a mean $A D C$ value of $0.76 \pm 0.19 \times 10^{-3} \mathrm{~mm}^{2} / \mathrm{s}$.

Conclusions: The present study shows clinical and imaging features of MS based upon a large patient sample in a multicenter design. MS occurs in most cases meta-chronous to the hematological disease and most commonly affects the cutis. One fourth of cases were identified incidentally on imaging, which needs awareness of the radiologists for possible diagnosis of MS.

Keywords: Myeloid sarcoma, Granulocytic sarcoma, Chloroma, Acute myeloid leukemia

\section{Background}

Myeloid sarcoma (MS), also known as granulocytic sarcoma or chloroma, is a solid extramedullary tumor originating from malignant primitive myeloid cells [1-3]. Its occurrence is linked to leukemic diseases of the myeloid cell line, most commonly in acute myeloid leukemia (AML) and less commonly in chronic myeloid leukemia

\footnotetext{
* Correspondence: Hans-jonas.meyer@medizin.uni-leipzig.de

${ }^{1}$ Department of Diagnostic and Interventional Radiology, University of Leipzig, University Hospital Leipzig, Liebigstraße 20, 04103 Leipzig, Germany Full list of author information is available at the end of the article
}

(CML), myelodysplastic syndrome (MDS) or other myeloproliferative disorders [4].

Most frequently, it is a recurrence of the primary disease, less commonly concurrently with the primary disease or it can even be the initial manifestation of the hematological disease, which can impose great diagnostic challenges $[4,5]$. A very rare form is the primary extramedullary manifestation of AML with normal appearing bone marrow, which comprises less than $1 \%$ of all patients with AML [6].

(c) The Author(s). 2019 Open Access This article is distributed under the terms of the Creative Commons Attribution 4.0 International License (http://creativecommons.org/licenses/by/4.0/), which permits unrestricted use, distribution, and reproduction in any medium, provided you give appropriate credit to the original author(s) and the source, provide a link to the Creative Commons license, and indicate if changes were made. The Creative Commons Public Domain Dedication waiver (http://creativecommons.org/publicdomain/zero/1.0/) applies to the data made available in this article, unless otherwise stated. 
An identified risk factor for MS is allogenic bone marrow transplantation [7]. The overall survival of patients with MS is poor with a median survival of 12.8 months [6].

MS can possibly occur in any organ system, which leads to a great amount of differential diagnosis, including other benign and malignant tumors, hematomas and inflammatory diseases depending on the localization $[2,4,8]$.

Currently, the body of literature investigating radiological findings of MS is composed of small patient samples based upon single center studies with inherent limitations [9-11].

Therefore, the purpose of this study was to evaluate clinical and imaging characteristics of MS based upon a large patient sample in a multicenter design.

\section{Methods}

\section{Patient sample}

The institutional ethic committee waived the need for informed consent due to the retrospective nature of the study (Committee of the University of Leipzig, Study codes Nr. 027/2002 and 162/2004). No permission was required to review the patient records. The radiology databases of 4 German university hospitals (University of Leipzig, Martin-Luther University of Halle (Saale), University Medicine of Göttingen, Ulm University Medical Center) were retrospectively screened for myeloid sarcoma in the time period between 01/2001 and 06/2019. Cases were included into the study either with histopathological confirmation of MS $(n=109 / 151,72.2 \%$ of all included patients) or with clinical highly suspicious lesions of MS with histopathological confirmed of associated hematological disease $(n=42 / 151,27.8 \%$ of all included patients). The overall patient sample was comprised of 151 patients with a median age of $55.5 \pm 15.1$ years (range 16-86 years). There were 76/151 female patients (50.3\%) and 75/151 (49.7\%) male patients.

\section{Clinical features}

The patient records were center specific reviewed. The underlying hematological disease was categorized in following subgroups: AML, CML, myeloproliferative syndrome, not further classified (MPS), MDS, primary idiopathic myelofibrosis, and chronic myelomonocytic leukemia (CMML). The kind of first clinical manifestation was evaluated and sorted as followed: neurological deficit; incidental finding by imaging; pain; skin-related color changes or swelling; organ-specific dysfunction.

\section{Image analysis}

For $57 / 151$ patients $(37.7 \%)$ computed tomography (CT) images and for $54 / 151$ patients (35.8\%) magnetic resonance imaging (MRI) were available.
Different CT scanners (Somatom Sensation 64, Somatom Definition AS 128 Siemens, Erlangen, Germany; Ingenuity and Brilliance iCT 256, Philips Medical Systems, Cleveland, OH, USA) were used. CT scans included cervical, thoracic, abdominal, and pelvic regions. In all patients $1.5 \mathrm{ml}$ of iodinated intravenous contrast medium per body mass were given at a rate of $1.5-2.5 \mathrm{ml} / \mathrm{s}$ by a power injector, with a scan delay of approximately $90 \mathrm{~s}$ (portal venous phase) after onset of injection.

For $2 / 151$ patients (1.3\%) $\mathrm{F}^{18}$-fluorodexyglucose(FDG-PET)-Positron-emission tomography (Siemens Biograph 16, Siemens Medical Solutions, Erlangen, Germany) was available.

For MRI, the following scanners were used: 3.0 T Magnetom Trio, $1.5 \mathrm{~T}$ Magnetom Vision Sonata Upgrade, 1.5 T Magnetom Aera, Magnetom Skyra 3.0 T, Magnetom Avanto 1.5 T (Siemens Medical Solutions, Erlangen, Germany).

All imaging studies were performed in clinical routine with routine protocols.

The imaging studies were re-evaluated by the boardcertified radiologists of every study center in awareness of the diagnosis. Following features were evaluated: type of modality (CT, MRI), maximum size (largest diameter in $\mathrm{mm}$, defined on the slide with the largest tumor appearance), number of lesions, localization, imaging appearance (hypodense/hypointense, isodense/isointense, hyperdense/hyperintense in comparison to surrounding muscle tissue) and type of enhancement (no enhancement; homogenous or heterogeneous enhancement).

\section{Statistical analysis}

Collected data were analyzed by means of descriptive statistics (absolute and relative frequencies) with SPSS (SPSS 25.0, SPSS Inc., Chicago IL, USA). Continuous variables were expressed as means \pm standard deviation (SD), and categorical variables as percentages. MannWhitney test was used for group comparisons.

\section{Results}

\section{Clinical findings}

Overall, 95/151 MS patients (62.9\%) suffered from primary AML, 29/151 (19.2\%) from secondary AML, (26 from MDS and 3 MPS), 18/151 (11.9\%) from CML, 3/ 151 (2.0\%) from MDS, 3/151 (2.0\%) from CMML, 2/151 (1.3\%) from MPS, and $1 / 151$ case $(0.7 \%)$ from primary idiopathic myelofibrosis.

In $15 / 151$ cases $(9.9 \%)$, the manifestation of MS preceded the systemic hematological disease. In 43/ 151 cases (28.4\%) first presentation of MS occurred simultaneously with the initial diagnosis of leukemia, and $92 / 151(60.9 \%)$ patients presented MS after the initial diagnosis. 
In $37 / 151$ patients $(24.5 \%)$, the lesions were detected incidentally by imaging. Clinically, typical cutaneous lesions were detected in 35/151 cases (23.2\%). Other leading symptoms were pain $(n=28 / 151,18.5 \%)$, neurological deficit $(n=27 / 151,17.9 \%)$, swelling $(n=$ $14 / 151,9.3 \%)$ and dysfunction of the affected organ $(n=10 / 151,6.0 \%)$.

\section{Localizations of MS}

In $128 / 151$ patients (84.8\%), MS was limited to one localization. Two different localizations occurred in 17/ 151 patients $(11.3 \%)$, three in $3 / 151$ patients $(2.0 \%)$ and four localizations in $3 / 151$ patients (2.0\%).

Most commonly, cutis was affected $(n=30 / 151$, $16.6 \%)$, followed by bone $(n=29 / 151,16.0 \%)$ and lymphatic tissue $(n=21 / 151,11.4 \%)$. Other localizations were rare (Table 1$)$.

\section{Imaging findings}

Figures 1, 2, 3 and 4 display typical imaging features of MS. Tumor size varied from $2 \mathrm{~mm}$ to $140 \mathrm{~mm}$ with a mean value of $37.5 \pm 25.3 \mathrm{~mm}$.

There were no statistically significant differences for tumor size in regard of the hematological disease (for AML mean value $37.9 \pm 26.6 \mathrm{~mm}$, for CML $32.4 \pm 18.7$, for secondary AML $42.7 \pm 22.7 \mathrm{~mm}, p=0.28$ ).

There was no difference in tumor size between incidental lesions and symptomatic lesions $(33.8 \pm 33.6 \mathrm{~mm}$ versus $38.8 \pm 25.2, p=0.41$ ).

CT

For 57/151 patients (37.7\%) CT images were available. Every lesion showed a moderate contrast enhancement after intravenous contrast media application. The contrast enhancement was homogenous in 40/57 patients (70.2\%), and inhomogeneous in $17 / 57$ patients (29.8\%).

\section{MRI}

For 54/151 patients (35.8\%) MRI was available. On T2weighted images, 7/54 lesions (13.0\%) were hypointense, 6/54 lesions (11.1\%) were isointense, and 41/54 (75.9\%) were hyperintense in comparison to the adjacent muscle tissue.

On T1-weighted images, MS were hypointense in 22/ 54 cases $(40.7 \%)$, isointense in $30 / 54$ cases $(55.6 \%)$ and hyperintense in $2 / 54$ cases $(3.7 \%)$ compared to the adjacent muscle tissue.

In $52 / 151$ cases $(34.4 \%)$ intravenous contrast media was applied. Most commonly a moderate homogenous enhancement was shown in $31 / 52$ cases (59.6\%). In $20 / 52$ cases $(38.5 \%)$ an inhomogeneous enhancement was detected. Only $1 / 52$ case (1.9\%) showed no enhancement.
Table 1 The identified localizations of Myeloid sarcoma. Twenty-three patients had 2 or more localizations resulting in overall 183 different lesions of Myeloid sarcoma in 26 different localizations

\begin{tabular}{|c|c|c|}
\hline Localization & Lesion number $(\mathrm{N})$ & Percent (\%) \\
\hline Skin & 30 & 16.6 \\
\hline Bone & 29 & 16.0 \\
\hline Lymphatic tissue & 21 & 11.4 \\
\hline Breast & 17 & 9.3 \\
\hline Central nervous system (brain) & 17 & 9.3 \\
\hline Muscle & 13 & 7.2 \\
\hline Central nervous system (spine) & 8 & 4.4 \\
\hline Orbita & 5 & 2.7 \\
\hline Central nervous system (meningeal) & 3 & 1.6 \\
\hline Testis & 3 & 1.6 \\
\hline Paranasal sinus & 3 & 1.6 \\
\hline Vagina & 3 & 1.6 \\
\hline Larynx & 3 & 1.6 \\
\hline Kidney & 2 & 1.1 \\
\hline Pancreas & 2 & 1.1 \\
\hline Pleural & 2 & 1.1 \\
\hline Salivary gland & 2 & 1.1 \\
\hline Stomach & 2 & 1.1 \\
\hline Cardiac & 2 & 1.1 \\
\hline Uterus & 2 & 1.1 \\
\hline Pharynx & 2 & 1.1 \\
\hline Bladder & 1 & 0.5 \\
\hline Peritoneal & 1 & 0.5 \\
\hline Pulmonary & 1 & 0.5 \\
\hline Ovarian & 1 & 0.5 \\
\hline Liver & 1 & 0.5 \\
\hline All & 183 & 100 \\
\hline
\end{tabular}

\section{Diffusion weighted imaging (DWI)}

Diffusion weighted imaging (DWI) was available for 17/151 patients (11.2\%). Different b-values were used according to study center and investigated body region. On ADC maps the signal intensity was in most lower in comparison to the adjacent muscle tissue ( $n=14 / 17,82.4 \%)$. Quantified, the mean apparent diffusion coefficient (ADC) value was $0.76 \pm 0.19 \times 10^{-3} \mathrm{~mm}^{2} / \mathrm{s}$, range $0.42-2.4 \times 10^{-3} \mathrm{~mm}^{2} / \mathrm{s}$.

Finally, $2 / 151$ patients $(1.3 \%)$ were investigated with FDG-PET. The lesions showed high elevated traceruptake.

\section{Discussion}

The present study provides clinical and imaging findings of MS based upon a large patient sample in a multicenter design. 


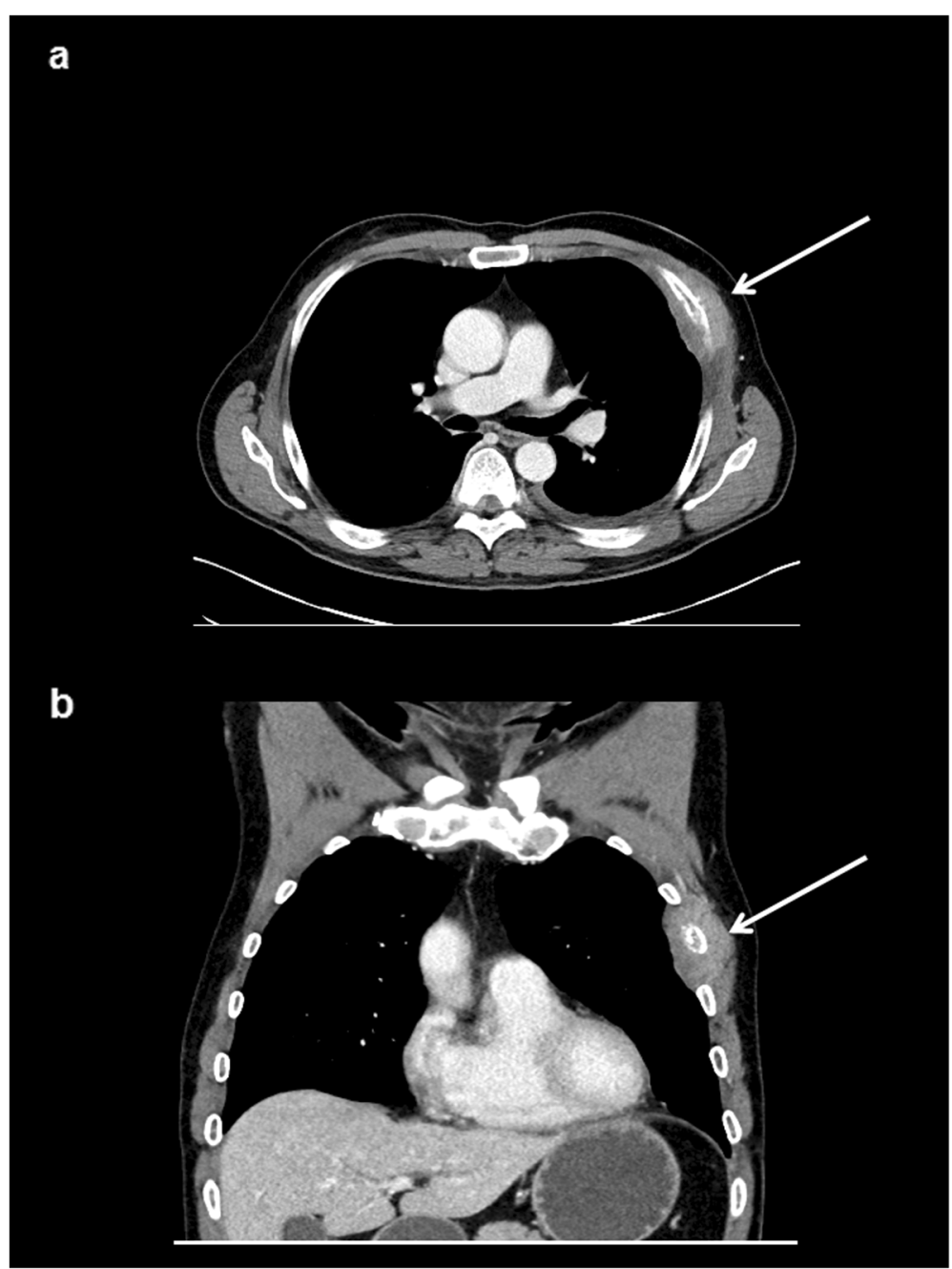

Fig. 1 Myeloid sarcoma of the chest wall in a male patient with known acute myeloid leukemia in a relapse setting. Contrast enhanced computed tomography in axial (a) and coronal plane (b) showing a relatively homogenous of the third left rip with an infiltration of the adjacent intercostal muscles (arrows)

MS most commonly occurs in patients with AML with a reported incidence of $2.5-9 \%$ [2, 4]. Furthermore, MS can less commonly occur in other hematological diseases, such as CML, MDS and myeloproliferative diseases with yet no reported systematic data regarding incidences. Concordantly, only few patients with these entities were identified in our patient sample.

Making the diagnosis of MS can be a challenge, especially in patients with extramedullary MS and normal appearing bone marrow as a first manifestation of AML [6]. These patients represent, however, less than $1 \%$ of all AML cases [6]. MS is associated with an overall poor survival with reported median survival of 12.8 to 15.9 months $[6,8,12]$. In short, sole presence of MS indicates poor outcome irrespective of the clinical setting.

\section{Clinical findings}

Principally, MS can be classified into 4 groups according to the manifestation. First is the manifestation of MS with concurrent acute myeloid leukaemia. In such cases the diagnosis of MS might be easy to make [5, 12, 13]. Secondly, extramedullary relapse of AML, including in the setting after bone marrow transplantation. Thirdly, blast phase/transformation of a myeloproliferative neoplasm or chronic myelomonocytic leukaemia $[5,12,13]$. Lastly, isolated MS, which occurs in association with a normal bone marrow biopsy and blood analysis, and in the absence of any history of myeloid neoplasia [6]. These cases of MS might be most challenging in diagnosis and needs histopathological evaluation of the MS suspicious lesion $[6,12,13]$.

The identified frequencies in the present study are well comparable with the literature. Most common 


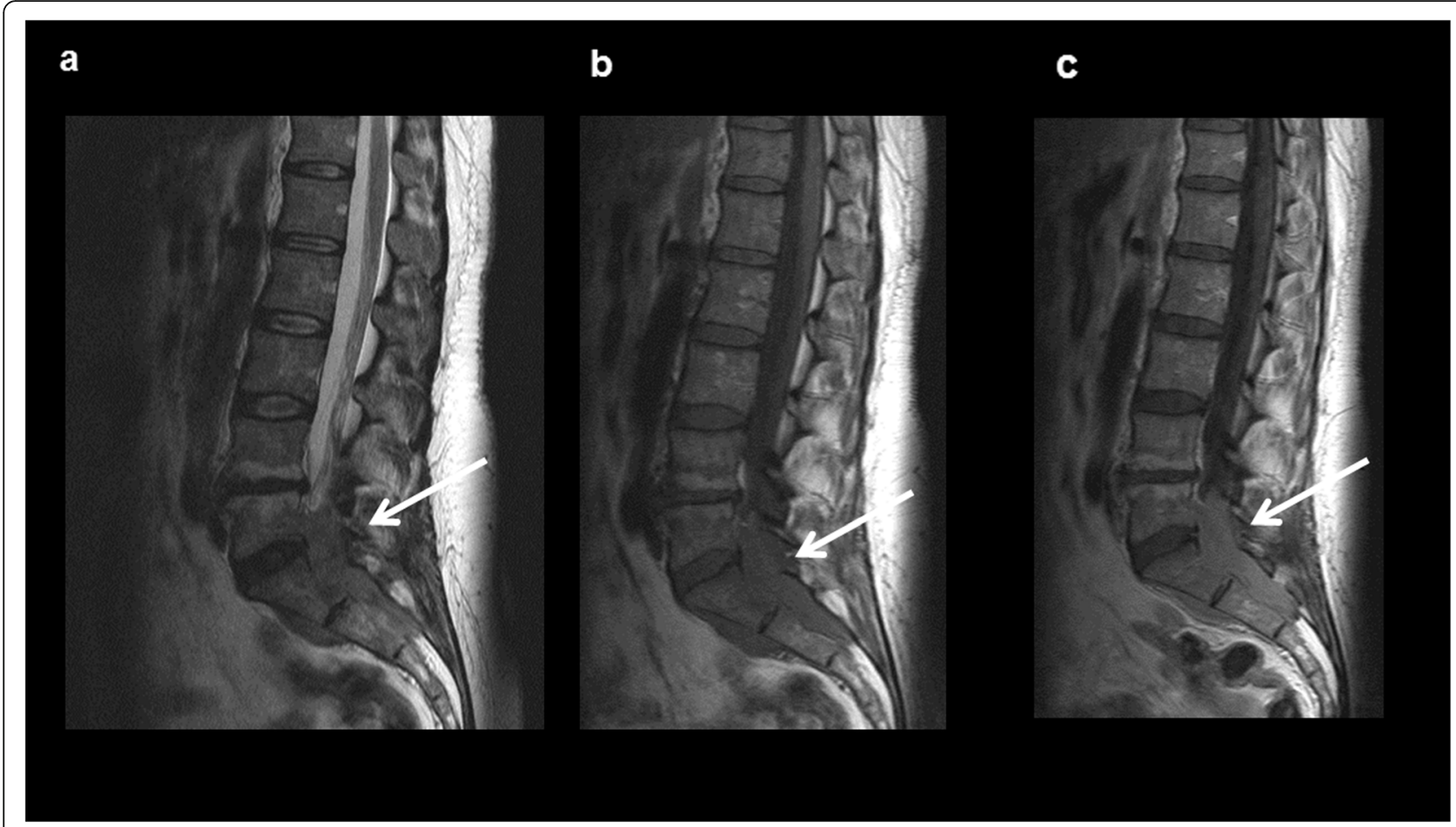

Fig. 2 Intraspinal manifestation of Myeloid sarcoma in the lumbosacral region in a female patient with known acute myeloid leukemia in a relapse setting. a On sagittal T2-weighted image, the lesion is homogenous, hyperintense compared to adjacent muscle tissue (arrow). b On sagittal T1-weighted image, the lesion is hypointense (arrow). c On sagittal T1- weighted image after application of contrast medium a strong homogenous contrast enhancement can be appreciated (arrow)

the extramedullary relapse setting of AML with up to $60 \%$, followed by simultaneous manifestation in $30 \%$ and less frequently, as an extramedullary manifestation of AML without bone morrow involvement in only few cases.
The diagnosis of MS can be challenging, and relatively high misdiagnosis rates have been reported varied from 25 to $47 \%$, which especially concern de novo manifestations without bone marrow involvement [12-14]. Possible misdiagnoses comprised Hodgkin-Lymphomas,

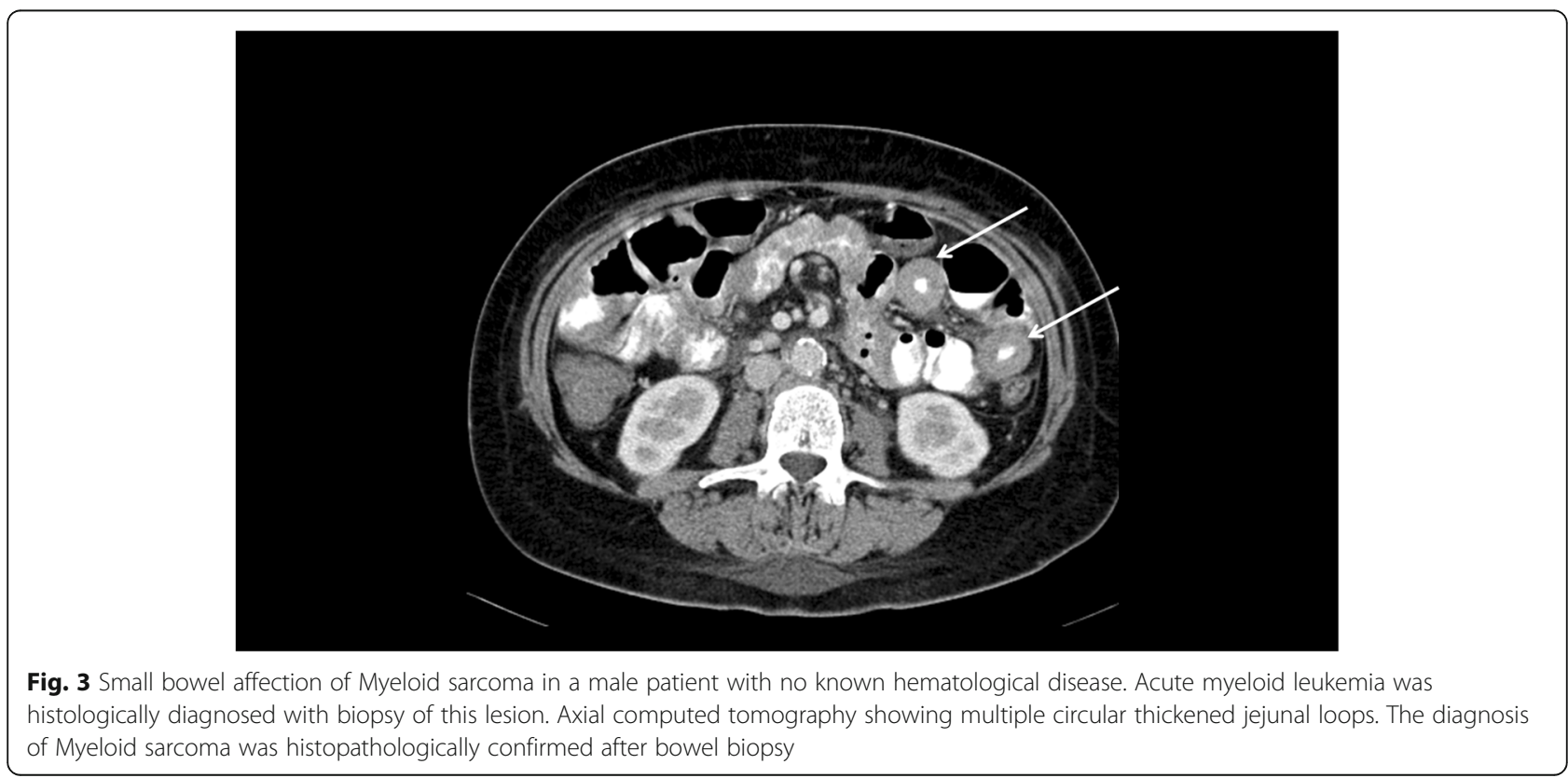




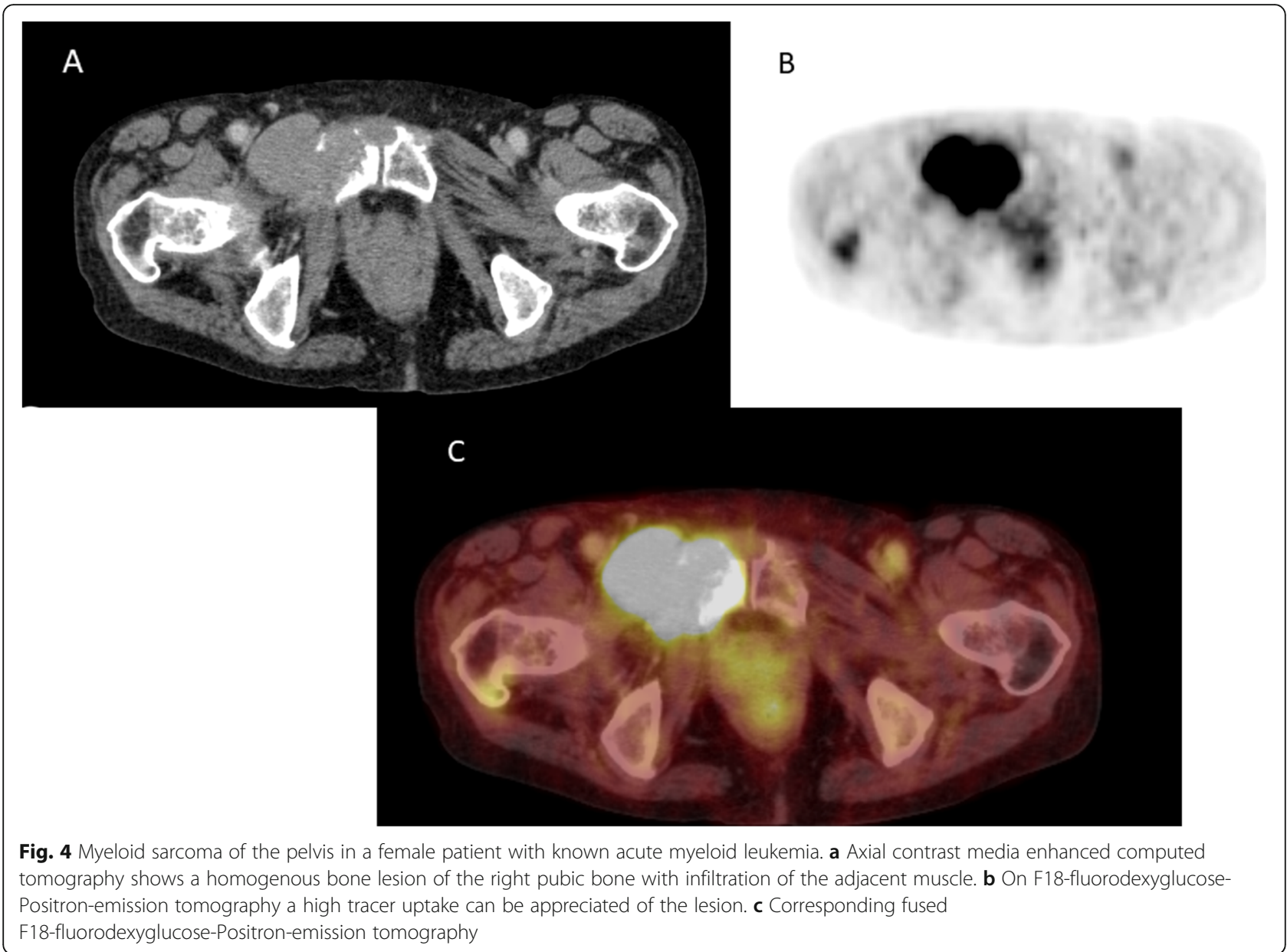

MALT lymphoma, or Ewing's sarcoma, which can show similar histopathologic characteristics [12].

Clinically, infectious diseases such as abscesses or hematomas should be considered as a possible differential diagnosis because these occur very frequently in leukemic patients with immunosuppression and thrombocytopenia, either due to chemotherapy or due to malignancy itself [8].

Regarding gender, a slight male predominance was identified in the literature $[6,13]$. However, in the present sample there was no gender predominance.

Clinical presentation of MS largely depends on the affected site. Correspondingly, MS can present with various symptoms, such as tumor mass effect or local organ dysfunction [2]. However, according to the literature, about half of patients with MS were asymptomatic and, therefore, they were identified by imaging [8]. Notably, in the present study, most cases of MS (24.5\%) were detected incidentally by imaging studies, which is caused by increasing usage of cross-sectional imaging in hematological patients. Therefore, the radiologist and oncologist need to be aware of MS.
Regarding localizations, MS affects most frequently cutis, subcutis and lymph nodes [5, 9, 13]. However, the frequencies of different localizations varied in several studies. In fact, Kaur et al. reported a skin involvement in up to $69.5 \%$ of patients in a case series of 22 patients [5]. Contrary, Pileri et al. reported a skin manifestation in only $28.2 \%$ of patients based upon 74 patients, which was yet the most common localization [13]. Kawamoto et al. reported clinicopathological findings of $131 \mathrm{pa}-$ tients with MS and identified that lymph nodes were the most frequent manifestation (55\%) [3]. Recently, a frequent involvement of the visceral soft tissue $(29.9 \%)$ as the most common localization was described [9]. MS can principally manifest in every organ resulting in rare organ manifestations including pulmonary, kidney, vaginal and uterine [8]. In the present study, most commonly cutis involvement was found, followed by bone and lymphatic tissue.

A relatively high amount of breast manifestations was identified in our patient sample. Contrary, in previous patient samples no breast manifestation was reported. This might be caused by a university hospital included 
in the study with a large breast center and therefore possible selection bias.

The affection of the central nervous system is rare with a reported frequency of $1.5 \%$, which can have a crucial impact on the clinical course of patients due to early neurological deficits [3]. We identified a higher rate, namely $9.3 \%$ of all acquired cases. Presumably, this might be caused by an increasing use of cross-sectional imaging in oncologic patients, which leads to more incidentally detected lesions. This might explain differences to older case series with lower reported frequency [15].

\section{Imaging findings}

Previously, only few reports with relatively small numbers of patients/lesions reported imaging findings in MS [9-11, 16, 17]. So far, Shinagare et al. described MRI features of 25 patients with 41 different MS localizations [16]. The authors reported that the lesions had a mean size of $5.6 \mathrm{~cm}$ (range $1-20 \mathrm{~cm}$ ), which is slightly higher than in our observation. Presumably, the results differ slightly due to the fact that in the mentioned study only lesions with MRI were included, whereas in the present study mostly CT was used. A MS lesion investigated by MRI might be a clinical symptomatic lesion with consecutive a larger size, whereas CT more commonly detects incidental lesions, which might be smaller in size.

CT findings of MS were reported to be variable, depending on the site of involvement [8]. Most commonly, on CT images, MS lesions were reported to be isodense to adjacent muscle tissue [9]. In contrast, cerebral manifestations were reported to be slightly hyperdense compared to the bordering brain [17]. After application of contrast medium, in most cases a homogenous enhancement was observed [9]. The homogenous CT-texture might reflect the histopathology and help to distinguish other malignant tumors with more necrotic areas. However, studies are needed to employ this imaging feature for discrimination purposes.

The present study corroborates the previous results with overall good comparable frequencies in regard of contrast media characteristics and density of CT images.

Regarding MRI, it was reported that $75.6 \%$ of MS lesions were isointense and $24.4 \%$ were hypointense on T1weighted images [16]. On T2-weighted images, 95.1\% were hyperintense and $4.9 \%$ were isointense [16]. In a recent study based on 28 patients, on T2-weighted images most cases $(82.1 \%)$ were hyperintense, whereas on T1weighted images $60.7 \%$ of the identified cases were isointense compared to adjacent muscle tissue [9]. Similar results were also reported in another case series [17].

Our results based on overall 54 lesions suggested similar frequencies in regard of signal intensities.

A widely used imaging technique is DWI, which can quantify proton movement in tissues and is, therefore, able to reflect microstructure of tissues [18]. It was previously reported that DWI is very sensitive for lymphoma lesions, which show a comparable histopathology characteristic as MS lesions [19]. So, it was reported that most MS lesions showed a diffusion restriction with up to $96 \%$ of cerebral lesions [10]. Similar results were identified based upon 10 cases with a more advanced imaging protocol [20]. Moreover, the ADC value increases after therapy [20]. In the present study, the mean ADC value was slightly higher than reported, yet with most a diffusion restriction pattern. This is most likely caused by a high cellularity of the lesions, as it was extensively investigated that $\mathrm{ADC}$ values are inversely correlated with tissue cellularity [18]. DWI might be a useful diagnostic tool for treatment evaluation of MS, which needs, however, more data. In regard of differential diagnosis, however, other malignant tumors and abscesses can also show restricted diffusion, which might reduce the diagnostic value of this sequence in clinical routine [21].

A beneficial imaging modality is FDG-PET/CT, which has a better accuracy than CT in diagnosing MS [2224]. As reported previously, MS lesions show typically an intense FDG uptake. More interestingly, the tracer uptake changed under therapy, which correlated with clinical outcome [22-24]. Moreover, FDG-PET can detect additional lesions, which were not clinically known [24]. Yet, systematic data are still needed for this imaging modality to prove possible benefit. Only few lesions in the present study were staged with PET/CT, which also showed an elevated FDG uptake in good agreement with the literature.

There are several limitations of the present study to address. Firstly, it is a retrospective study with known possible inherent bias. Secondly, the patient sample is recruited from 4 German university hospitals with possible different diagnosis and treatment regimens and imaging studies were not available for all patients. Thus, due to the multicenter design the present patient sample is relatively large. Furthermore, multicenter prospective studies are difficult to perform due to low incidence of MS. Thirdly, the imaging analysis was performed in the involved centers and no central reading was performed, which might harbor some possible differences in regard of imaging assessment. However, the reading was performed by board certified radiologists and can, therefore, be generalized for clinical routine. Fourthly, the diagnosis of MS was not histopathologically confirmed in all cases. It has been reported that the misdiagnosis rate of MS is up to 47\% [2], which might be substantial lower in the present patient sample because only clinical highly suspicious MS-lesions were included in the analysis.

The oncologist and radiologist need to keep in mind the diagnosis of MS for every unclear and new occurrent 
lesion in a patient with known AML, independently from the localization.

\section{Conclusions}

Myeloid sarcoma is a rare manifestation of several hematological malignancies, most common in AML, which can affect any part of the body. Most commonly, it manifests within the skin and bone, followed by lymphatic tissue. This multicenter retrospective study provides clinical and imaging findings based upon a large patient sample.

\section{Abbreviations \\ ADC: Apparent diffusion coefficient; AML: Acute myeloid leukemia; CML: Chronic myeloid leukemia; CMML: Chronic myelomonocytic leukemia; CNS: Central nervous system; CT: Computed tomography; DWI: Diffusion weighted imaging; FDG-PET: Fluorodeoxyglucose-Positron-emission tomography; MDS: Myeloid dysplastic syndrome; MPS: Myeloid proliferative syndrome; MRI: Magnetic resonance imaging; MS: Myeloid sarcoma}

\section{Acknowledgements}

None.

\section{Authors' contributions}

HJM and AS participated in design, data collection, data analysis, and data interpretation. HJM and AS drafting of the final manuscript. SAS, SW, DS, FB, WP data analysis and interpretation All authors have read and approved the final manuscript.

\section{Funding}

This research did not receive any specific grant from funding agencies in the public, commercial, or not-for-profit sectors.

\section{Availability of data and materials}

The datasets used and/or analysed during the current study available from the corresponding author on reasonable request.

\section{Ethics approval and consent to participate}

All procedures performed in the study involving human participants were in compliance with the Helsinki Declaration and University of Leipzig. Written informed consent was waived due to the retrospective study design.

\section{Consent for publication}

Not applicable.

\section{Competing interests}

The authors declare that they have no competing interests.

\begin{abstract}
Author details
${ }^{1}$ Department of Diagnostic and Interventional Radiology, University of Leipzig, University Hospital Leipzig, Liebigstraße 20, 04103 Leipzig, Germany. ${ }^{2}$ Department of Hematology and Oncology, University Hospital Leipzig, Leipzig, Germany. ${ }^{3}$ Department of Diagnostic and Interventional Radiology, Ulm University Medical Center, 89081 Ulm, Germany. ${ }^{4}$ Department of Diagnostic and Interventional Radiology, University Medicine Göttingen, 37075 Göttingen, Germany. ${ }^{5}$ Department of Hematology and Medical Oncology, University Medicine Göttingen, 37075 Göttingen, Germany. ${ }^{6}$ Department of Diagnostic and Interventional Radiology, University Hospital of Halle (Saale), 06097 Halle (Saale), Germany.
\end{abstract}

Received: 12 October 2018 Accepted: 12 November 2019 Published online: 27 November 2019

\section{References}

1. Liu Pl, Ishimaru T, McGregor DH, Okada H, Steer A. Autopsy study of granulocytic sarcoma (chloroma) in patients with myelogenous leukemia, Hiroshima-Nagasaki 1949-1969. Cancer. 1973;31(4):948-55.
2. Almond LM, Charalampakis M, Ford SJ, Gourevitch D, Desai A. Myeloid sarcoma: presentation, diagnosis, and treatment. Clin Lymphoma Myeloma Leuk. 2017;17(5):263-7.

3. Kawamoto K, Miyoshi H, Yoshida N, Takizawa J, Sone H, Ohshima K Clinicopathological, cytogenetic, and prognostic analysis of 131 myeloid sarcoma patients. Am J Surg Pathol. 2016;40(11):1473-83.

4. Campidelli C, Agostinelli C, Stitson R, Pileri SA. Myeloid sarcoma: extramedullary manifestation of myeloid disorders. Am J Clin Pathol. 2009;132(3):426-37.

5. Kaur V, Swami A, Alapat D, Abdallah AO, Motwani P, Hutchins LF. Clinical characteristics, molecular profile and outcomes of myeloid sarcoma: a single institution experience over 13 years. Hematology. 2017;23(1):17-24.

6. Goyal G, Bartley AC, Patnaik MM, Litzow MR, Al-Kali A, Go RS. Clinical features and outcomes of extramedullary myeloid sarcoma in the United States: analysis using a national data set. Blood Cancer J. 2017;7(8):e592.

7. Mortimer J, Blinder MA, Schulman S, Appelbaum FR, Buckner CD, Clift RA. Relapse of acute leukemia after marrow transplantation: natural history and results of subsequent therapy. J Clin Oncol. 1989;7(1):50-7.

8. Singh A, Kumar P, Chandrashekhara SH, Kumar A. Unravelling chloroma: review of imaging findings. Br J Radiol. 2017;90(1075):20160710.

9. Meyer HJ, Beimler M, Borte G, Pönisch W, Surov A. Radiological and clinical patterns of myeloid sarcoma. Radiol Oncol. 2019;53(2):213-8.

10. Chaudhry AA, Gul M, Chaudhry AA, Dunkin J. Qualitative assessment of diffusion weighted imaging and susceptibility weighted imaging of myeloid sarcoma involving the brain. J Comput Assist Tomogr. 2016;40(1):61-6.

11. Choi EK, Ha HK, Park SH, Lee SJ, Jung SE, Kim KW, Lee SS. Granulocytic sarcoma of bowel: CT findings. Radiology. 2007;243(3):752-9.

12. Wilson CS, Medeiros L. Extramedullary manifestations of myeloid neoplasms. Am J Clin Pathol. 2015;144(2):219-39.

13. Pileri SA, Ascani S, Cox MC, Campidelli C, Bacci F, Piccioli M, et al. Myeloid sarcoma: clinico-pathologic, phenotypic and cytogenetic analysis of 92 adult patients. Leukemia. 2007;21(2):340-50.

14. Roth MJ, Medeiros LJ, Elenitoba-Johnson K, Kuchnio M, Jaffe ES, StetlerStevenson M. Extramedullary myeloid cell tumors: an immunohistochemical study of 29 cases using routinely fixed and processed paraffin-embedded tissue sections. Arch Pathol Lab Med. 1995;119(9):790-8.

15. Neiman RS, Barcos M, Berard C, Bonner H, Mann R, Rydell RE, Bennett JM. Granulocytic sarcoma: a clinicopathologic study of 61 biopsied cases. Cancer. 1981;48(6):1426-37.

16. Shinagare AB, Krajewski KM, Hornick JL, Zukotynski K, Kurra V, Jagannathan JP. MRI for evaluation of myeloid sarcoma in adults: a single-institution 10-year experience. AJR Am J Roentgenol. 2012;199(6):1193-8.

17. Ooi GC, Chim CS, Khong PL, Au WY, Lie AK, Tsang KW, Kwong YL. Radiologic manifestations of granulocytic sarcoma in adult leukemia. AJR Am J Roentgenol. 2011:176(6):1427-31.

18. Surov A, Meyer HJ, Wienke A. Correlation between apparent diffusion coefficient (ADC) and cellularity is different in several tumors: a metaanalysis. Oncotarget. 2017;8(35):59492-9.

19. Mayerhoefer ME, Karanikas G, Kletter K, Prosch H, Kiesewetter B, Skrabs C, et al. Evaluation of diffusion-weighted magnetic resonance imaging for follow-up and treatment response assessment of lymphoma: results of an 18F-FDG-PET/CT-controlled prospective study in 64 patients. Clin Cancer Res. 2015;21(11):2506-13.

20. Hou X, Du L, Yu H, Zhang X. Use of magnetic resonance imaging for diagnosis and after treatment of patients with myeloid sarcoma of the brain. Oncotarget. 2017;8(60):102581-9.

21. Noguchi K, Watanabe N, Nagayoshi T, Kanazawa T, Toyoshima S, Shimizu M Seto $\mathrm{H}$. Role of diffusion-weighted echo-planar MRI in distinguishing between brain brain abscess and tumour: a preliminary report. Neuroradiology. 1999:41(3):171-4.

22. Aschoff $P$, Häntschel M, Oksüz M, Werner MK, Lichy M, Vogel W, Pfannenberg C. Integrated FDG-PET/CT for detection, therapy monitoring and follow-up of granulocytic sarcoma. Initial results. Nuklearmedizin. 2009;48(5):185-91.

23. Lee EY, Anthony MP, Leung AY, Loong F, Khong PL. Utility of FDG PET/CT in the assessment of myeloid sarcoma. AJR Am J Roentgenol. 2012;198(5):1175-9.

24. Stölzel F, Röllig C, Radke J, Mohr B, Platzbecker U, Bornhäuser M, et al. ${ }^{18} \mathrm{~F}$ FDG-PET/CT for detection of extramedullary acute myeloid leukemia. Haematologica. 2011;96(10):1552-6.

\section{Publisher's Note}

Springer Nature remains neutral with regard to jurisdictional claims in published maps and institutional affiliations. 